\title{
Residue Network Involved in the Allosteric Regulation of Cystathionine $\beta$-synthase Domain-containing Pyrophosphatase by Adenine Nucleotides
}

\author{
Viktor A. Anashkin ${ }^{\dagger}$, Anu Salminen ${ }^{\ddagger}$, Ekaterina Osipova $^{\dagger}$, Svetlana A. Kurilova ${ }^{\dagger}$, Ilia D. Deltsov ${ }^{\dagger}$, \\ Reijo Lahti ${ }^{\ddagger}$, and Alexander A. Baykov*,† \\ $\dagger$ Belozersky Institute of Physico-Chemical Biology and Department of Chemistry, Lomonosov Moscow \\ State University, Moscow 119899, Russia \\ $\ddagger$ Department of Biochemistry, University of Turku, FIN-20014 Turku, Finland
}



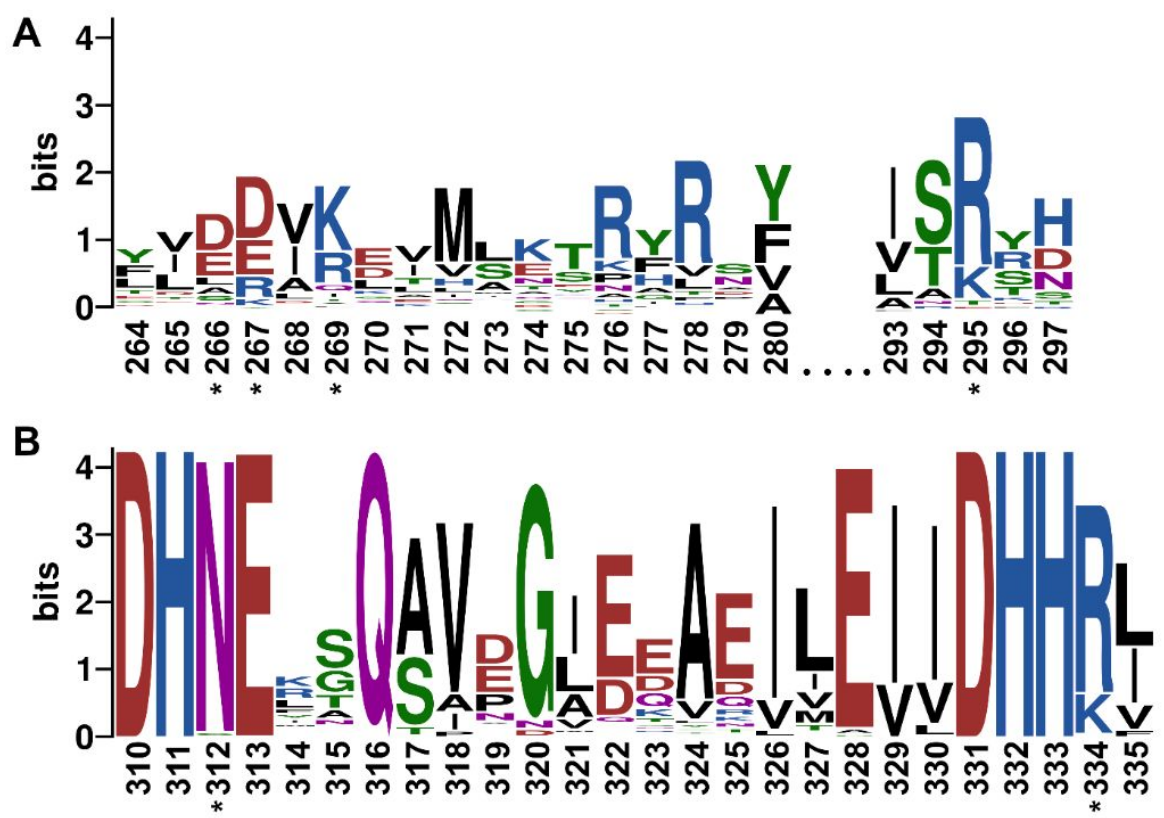

Figure S1. A WebLogo ${ }^{1}$ representation of the multiple sequence alignment of 188 CBS-PPases in the regions belonging to the $\mathrm{CBS} 2$ domain $(A)$ and $\mathrm{DHH}$ domain $(B)$. The residues presumably participating in signal transduction between the domains and selected for mutagenesis are marked by asterisk. 


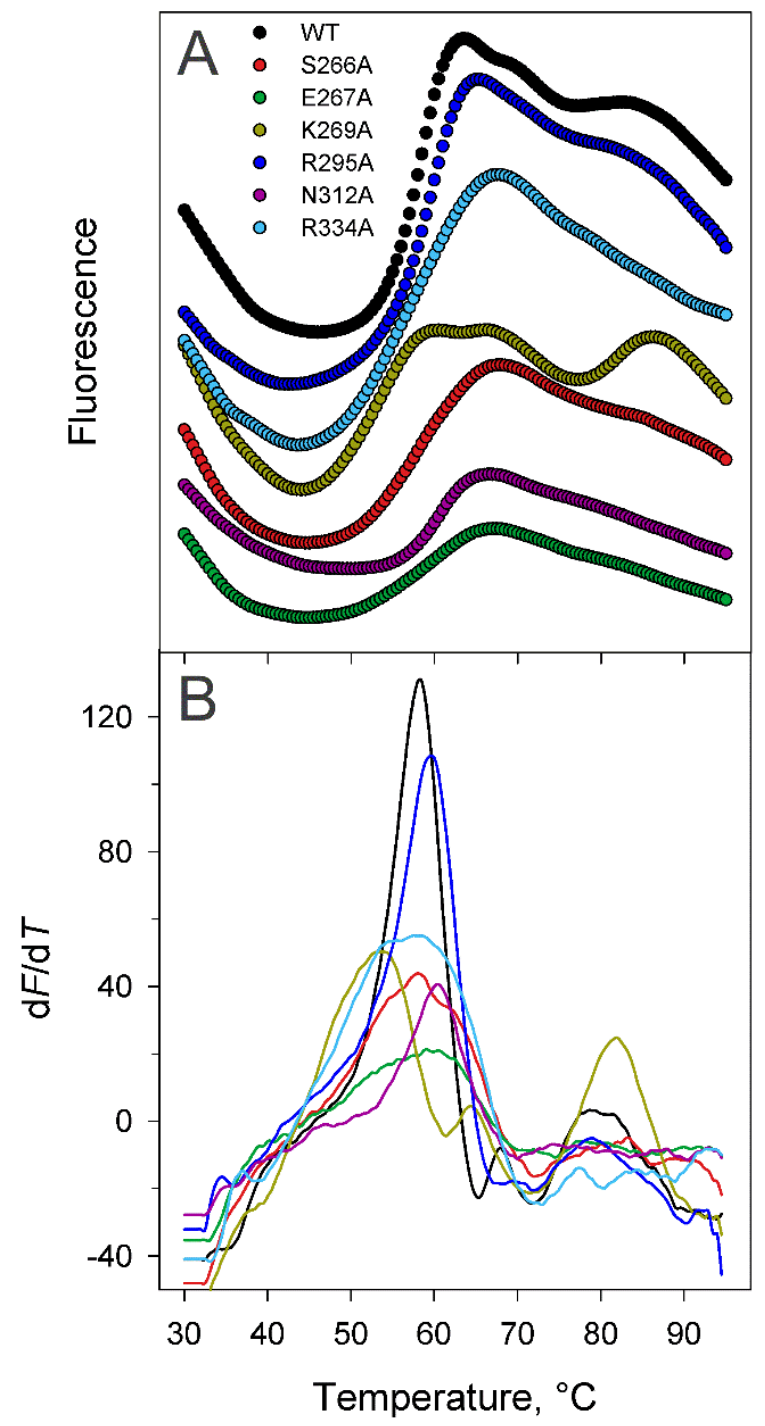

Figure S2. Initial Thermofluor data for $d h$ PPase variants $(A)$ and the first-derivative plots $(B)$. 

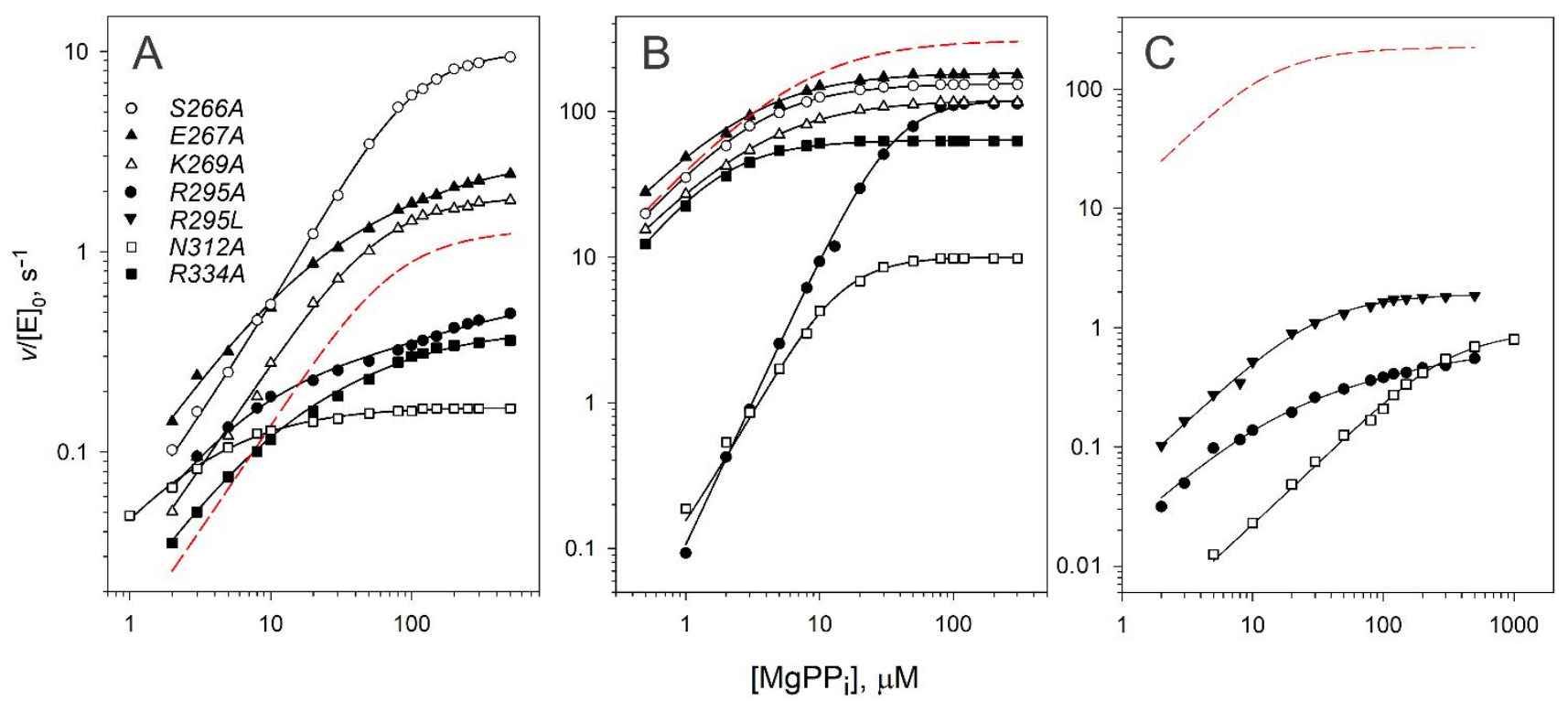

Figure S3. Dependences of the activities of $d h$ PPase alanine and leucine variants on substrate concentration in the presence AMP $(A), \operatorname{Ap}_{4} \mathrm{~A}(B)$ and ATP $(C)$ measured at $5 \mathrm{mM}$ free $\mathrm{Mg}^{2+}$. Nucleotide concentrations were as follows: AMP, $500 \mu \mathrm{M}$ with R334A and $50 \mu \mathrm{M}$ with all other variants; $\mathrm{Ap}_{4} \mathrm{~A}, 5 \mu \mathrm{M}$; ATP, $10 \mu \mathrm{M}$ with wild type enzyme and $100 \mu \mathrm{M}$ with all variant enzymes. The lines show the best fits of eqn (2). Symbols for all panels are defined in panel $A$. The red dashed curves refer to wild type $d h$ PPase and are taken from reference 2 (panel $B$ ) or 3 (panels $A$ and $C$ ).

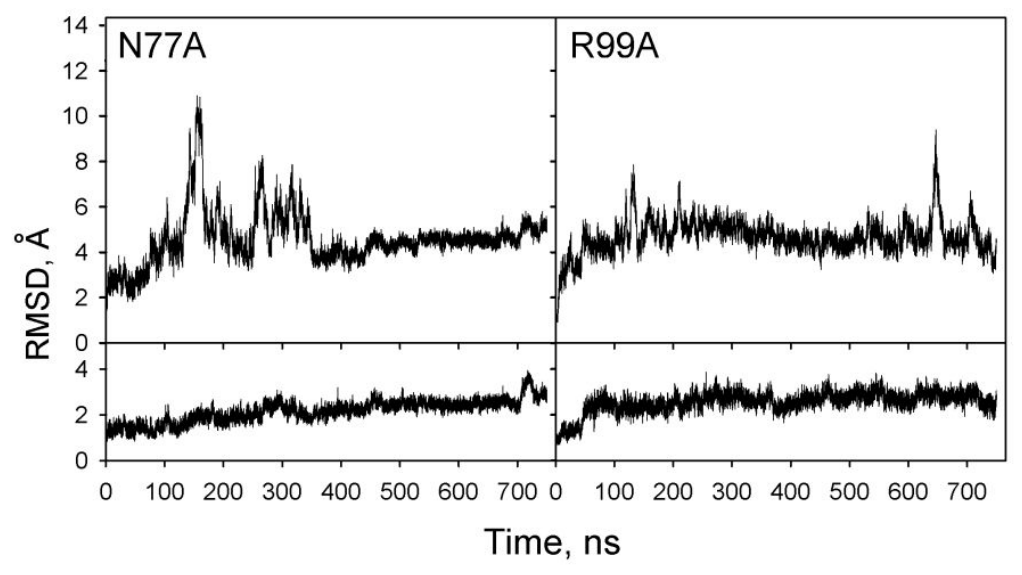

Figure S4. RMSD changes during MD simulations. RMSD values for variant $b s$ PPases versus the wild-type crystal structure are for the backbone atoms in the entire dimers (top panels) or in only two subunit interface-forming DHH domains (bottom panels). 
Table S1. Melting temperatures of $d h$ PPase alanine variants. Values were precise to $\pm 1^{\circ} \mathrm{C}$. Large effect is indicated by boldface.

\begin{tabular}{lc}
\hline Enzyme & $T_{\mathrm{m}},{ }^{\circ} \mathrm{C}$ \\
\hline WT & 57 \\
S266A & 58 \\
E267A & 61 \\
K269A & $\mathbf{5 4}$ \\
R295A & 60 \\
N312A & 60 \\
R334A & 57 \\
\hline
\end{tabular}

Table S2. Sedimentation data for $d h$ PPase alanine variants. The percentage of the dominant form was calculated by peak integration; the percentage of a lower-mass form did not exceed $5 \%$ in all cases. Large effects are indicated by boldface.

\begin{tabular}{lcc}
\hline Enzyme & $s_{20, \mathrm{w}}, \mathrm{S}$ & $\begin{array}{c}\text { Dominant } \\
\text { form, } \%\end{array}$ \\
\hline WT & 9.9 & 90 \\
S266A & 10.0 & 85 \\
E267A & 10.0 & 80 \\
K269A & $\mathbf{9 . 5}$ & 80 \\
R295A & 10.4 & 90 \\
N312A & $\mathbf{9 . 6}$ & 86 \\
R334A & 9.9 & 90 \\
\hline
\end{tabular}


Table S3. Parameter values in Scheme 2 for AMP inhibition of $d h P$ Pase variants measured at $50 \mu \mathrm{M}$ $\mathrm{MgPP}_{\mathrm{i}}$ and $5 \mathrm{mM}$ free $\mathrm{Mg}^{2+a}$. Large effects are indicated by boldface. The Hill coefficient, $h$, was estimated with the equation $v=v_{0}+\left(v_{\mathrm{N}}-v_{0}\right) /\left(1+K_{0.5} /[\mathrm{N}]^{h}\right)$, where $v$ is measured rate, $v_{0}$ and $v_{\mathrm{N}}$ are rates at zero and infinite nucleotide concentrations, respectively, and $[\mathrm{N}]$ is nucleotide concentration. Large effects are indicated by boldface.

\begin{tabular}{lcccccc}
\hline Enzyme & $A_{\mathrm{N}} / A_{0}$ & $K_{\mathrm{N} 1}, \mu \mathrm{M}$ & $K_{\mathrm{N} 2}, \mu \mathrm{M}$ & $\sqrt{ } K_{\mathrm{N} 1} K_{\mathrm{N} 2}, \mu \mathrm{M}$ & $K_{\mathrm{N} 1} / K_{\mathrm{N} 2}$ & $h$ \\
\hline Wild type & $0.062 \pm 0.013$ & $14 \pm 8$ & $0.25 \pm 0.15$ & $1.87 \pm 0.12$ & $48 \pm 10$ & $1.47 \pm 0.15$ \\
S266A & $0.047 \pm 0.003$ & $10 \pm 1$ & $1.3 \pm 0.2$ & $3.53 \pm 0.09$ & $8 \pm 2$ & $1.43 \pm 0.04$ \\
S266D & $0.030 \pm 0.002$ & $40 \pm 17$ & $0.12 \pm 0.05$ & $2.23 \pm 0.05$ & $>200$ & $1.87 \pm 0.05$ \\
E267A & $0.020 \pm 0.001$ & $19 \pm 2$ & $0.26 \pm 0.03$ & $2.20 \pm 0.04$ & $74 \pm 18$ & $1.75 \pm 0.04$ \\
K269A & $0.052 \pm 0.002$ & $5.5 \pm 0.3$ & $0.89 \pm 0.04$ & $2.21 \pm 0.02$ & $6.2 \pm 0.6$ & $1.41 \pm 0.02$ \\
K269R & $0.008 \pm 0.003$ & $3.6 \pm 0.6$ & $0.50 \pm 0.07$ & $1.39 \pm 0.06$ & $7 \pm 2$ & $1.38 \pm 0.05$ \\
K269E & $0.024 \pm 0.002$ & $>50$ & $<0.05$ & $1.62 \pm 0.05$ & $>\mathbf{1 0 0 0}$ & $2.00 \pm 0.08$ \\
R295A & $0.662 \pm 0.004$ & $\mathbf{0 . 0 7 4} \pm \mathbf{0 . 0 2}$ & $0.082 \pm 0.008$ & $\mathbf{0 . 0 7 8} \pm \mathbf{0 . 0 0 4}$ & $\mathbf{0 . 8 9} \pm \mathbf{0 . 1 7}$ & $\mathbf{0 . 9 8} \pm \mathbf{0 . 0 4}$ \\
R295L & $0.32 \pm 0.01$ & $\mathbf{0 . 2 0} \pm \mathbf{0 . 0 2}$ & $0.046 \pm 0.008$ & $\mathbf{0 . 0 9 7} \pm \mathbf{0 . 0 0 5}$ & $\mathbf{4 . 4} \pm \mathbf{1 . 5}$ & $1.32 \pm 0.07$ \\
R295E & $0.30 \pm 0.01$ & $70 \pm 6$ & $\mathbf{4 4} \pm \mathbf{4}$ & $\mathbf{5 5} \pm \mathbf{2}$ & $\mathbf{1 . 6} \pm \mathbf{0 . 3}$ & $1.10 \pm 0.04$ \\
N312A & $0.19 \pm 0.01$ & $>\mathbf{5 0 0}$ & $<0.5$ & $0.31 \pm 0.02$ & $>\mathbf{1 0 0 0}$ & $2.3 \pm 0.2$ \\
R334A & $0.047 \pm 0.014$ & $90 \pm 50$ & $1.4 \pm 0.8$ & $11 \pm 1$ & $70 \pm 80$ & $1.72 \pm 0.14$ \\
R334K & $0.010 \pm 0.003$ & $12 \pm 4$ & $0.23 \pm 0.08$ & $1.63 \pm 0.08$ & $50 \pm 40$ & $1.66 \pm 0.08$ \\
\hline
\end{tabular}

a AMP concentration was varied in the range of $0.001-500 \mu \mathrm{M}$. 
Table S4. Parameter values in Scheme 2 for $\mathrm{Ap}_{4} \mathrm{~A}$ activation of $d h \mathrm{PPase}$ variants measured at $50 \mu \mathrm{M}$ $\mathrm{MgPP}_{\mathrm{i}}$ and $5 \mathrm{mM}$ free $\mathrm{Mg}^{2+a}$. Large effects are indicated by boldface.

\begin{tabular}{lcccccc}
\hline Enzyme & $A_{\mathrm{N}} / A_{0}$ & $K_{\mathrm{N} 1}, \mathrm{nM}$ & $K_{\mathrm{N} 2}, \mathrm{nM}$ & $\sqrt{ } K_{\mathrm{N} 1} K_{\mathrm{N} 2}, \mathrm{nM}$ & $K_{\mathrm{N} 1} / K_{\mathrm{N} 2}$ & $h$ \\
\hline Wild type & $3.8 \pm 0.1$ & $5.0 \pm 0.4$ & $5.4 \pm 0.6$ & $5.3 \pm 0.2$ & $1.00 \pm 0.06$ & $0.98 \pm 0.04$ \\
S266A & $1.47 \pm 0.02$ & $10 \pm 2$ & $7 \pm 2$ & $8.8 \pm 0.9$ & $1.4 \pm 0.4$ & $1.08 \pm 0.11$ \\
S266D & $2.6 \pm 0.1$ & $1.4 \pm 0.3$ & $1.9 \pm 0.4$ & $1.6 \pm 0.2$ & $0.7 \pm 0.2$ & $0.95 \pm 0.08$ \\
E267A & $2.71 \pm 0.06$ & $2.6 \pm 0.3$ & $3.0 \pm 0.3$ & $2.8 \pm 0.1$ & $0.86 \pm 0.16$ & $0.98 \pm 0.04$ \\
K269A & $3.6 \pm 0.5$ & $29 \pm 2$ & $24 \pm 1$ & $27 \pm 6$ & $1.2 \pm 0.1$ & $1.04 \pm 0.05$ \\
K269R & $2.95 \pm 0.04$ & $2.9 \pm 0.2$ & $3.3 \pm 0.2$ & $3.1 \pm 0.1$ & $0.87 \pm 0.09$ & $0.97 \pm 0.03$ \\
K269E & $2.46 \pm 0.07$ & $1.6 \pm 0.2$ & $2.5 \pm 0.3$ & $2.0 \pm 0.2$ & $0.62 \pm 0.14$ & $0.93 \pm 0.06$ \\
R295A & $238 \pm 3$ & $177 \pm 6$ & $230 \pm 20$ & $191 \pm 8$ & $0.77 \pm 0.08$ & $0.99 \pm 0.02$ \\
R295L & $12.4 \pm 0.7$ & $5.4 \pm 0.4$ & $5.0 \pm 0.5$ & $5.2 \pm 0.2$ & $1.0 \pm 0.2$ & $1.04 \pm 0.04$ \\
R295E & $\mathbf{9 2} \pm \mathbf{1 0}$ & $\mathbf{1 2 0} \pm \mathbf{6}$ & $\mathbf{1 2 0} \pm \mathbf{1 3}$ & $\mathbf{1 2 1} \pm \mathbf{5}$ & $1.0 \pm 0.1$ & $1.03 \pm 0.03$ \\
N312A & $12 \pm 1$ & $\mathbf{1 3 0} \pm \mathbf{1 0}$ & $\mathbf{1 5 0} \pm \mathbf{1 0}$ & $\mathbf{1 3 8} \pm \mathbf{3}$ & $0.86 \pm 0.06$ & $0.97 \pm 0.02$ \\
R334A & $11 \pm 1$ & $\mathbf{5 2} \pm \mathbf{8}$ & $38 \pm 5$ & $45 \pm 2$ & $1.4 \pm 0.3$ & $1.07 \pm 0.05$ \\
R334K & $3.56 \pm 0.04$ & $5.1 \pm 0.3$ & $6.1 \pm 0.3$ & $5.6 \pm 0.1$ & $0.85 \pm 0.08$ & $0.96 \pm 0.02$ \\
\hline
\end{tabular}

${ }^{a} \mathrm{Ap}_{4} \mathrm{~A}$ concentration was varied in the range of $0.00002-20 \mu \mathrm{M}$. 
Table S5. Parameter values in Scheme 2 for ATP modulation of the activities of $d h$ PPase variants measured at $50 \mu \mathrm{M} \mathrm{MgPP}$ and $5 \mathrm{mM}$ free $\mathrm{Mg}^{2+a}$. Large effects are indicated by boldface.

\begin{tabular}{lcccccc}
\hline Enzyme & $A_{\mathrm{N}} / A_{0}$ & $K_{\mathrm{N} 1}, \mu \mathrm{M}$ & $K_{\mathrm{N} 2}, \mu \mathrm{M}$ & $\sqrt{ } K_{\mathrm{N} 1} K_{\mathrm{N} 2}, \mu \mathrm{M}$ & $K_{\mathrm{N} 1} / K_{\mathrm{N} 2}$ & $h$ \\
\hline Wild type $^{b}$ & $2.5 \pm 0.2$ & $4.6 \pm 1.6$ & $0.75 \pm 0.05$ & $1.9 \pm 0.2$ & $6 \pm 2$ & $1.45 \pm 0.13$ \\
R295A & $\mathbf{0 . 6 3} \pm \mathbf{0 . 0 1}$ & $\mathbf{0 . 2 0} \pm \mathbf{0 . 0 3}$ & $0.62 \pm 0.09$ & $0.34 \pm 0.03$ & $\mathbf{0 . 3 1} \pm \mathbf{0 . 0 7}$ & $\mathbf{0 . 7 6} \pm \mathbf{0 . 0 5}$ \\
R295L & $\mathbf{0 . 4 0} \pm \mathbf{0 . 0 1}$ & $\mathbf{0 . 3 5} \pm \mathbf{0 . 0 5}$ & $1.2 \pm 0.2$ & $0.64 \pm 0.07$ & $\mathbf{0 . 2 9} \pm \mathbf{0 . 0 7}$ & $\mathbf{0 . 7 5} \pm \mathbf{0 . 0 6}$ \\
N312A & $\mathbf{0 . 2 2} \pm \mathbf{0 . 0 2}$ & $6 \pm 2$ & $0.2 \pm 0.1$ & $1.0 \pm 0.1$ & $17 \pm 7$ & $1.54 \pm 0.01$ \\
\hline
\end{tabular}

${ }^{a}$ ATP concentration was varied in the range of $0.001-400 \mu \mathrm{M}$.

${ }^{b}$ From reference 3 ; the $K_{\mathrm{N}}$ values were converted to microscopic constants.

Table S6. Parameter values in Scheme 1 for $\mathrm{PP}_{\mathrm{i}}$ hydrolysis by $d h \mathrm{PPase}$ variants measured at $5 \mathrm{mM}$ free $\mathrm{Mg}^{2+a}$. Large effects are indicated by boldface. The Hill coefficient, $h$, was estimated with the equation $v=v_{\infty} /\left(1+K_{0.5} /[\mathrm{S}]^{h}\right)$, where $v$ is measured rate and $[\mathrm{S}]$ is substrate concentration. Large effects are indicated by boldface.

\begin{tabular}{lcccccc}
\hline Enzyme & $k_{\text {cat }}, \mathrm{s}^{-1}$ & $K_{\mathrm{m} 1}, \mu \mathrm{M}$ & $K_{\mathrm{m} 2}, \mu \mathrm{M}$ & $\sqrt{ } K_{\mathrm{m} 1} K_{\mathrm{m} 2}, \mu \mathrm{M}$ & $K_{\mathrm{m} 1} / K_{\mathrm{m} 2}$ & $h$ \\
\hline Wild type $^{b}$ & $175 \pm 10$ & $52 \pm 2$ & $5.0 \pm 0.5$ & $17 \pm 1$ & $10.3 \pm 0.4$ & $1.29 \pm 0.06$ \\
S266A & $150 \pm 10$ & $76 \pm 8$ & $25 \pm 6$ & $44 \pm 4$ & $3.0 \pm 0.9$ & $1.19 \pm 0.05$ \\
S266D & $162 \pm 5$ & $146 \pm 13$ & $21 \pm 4$ & $56 \pm 3$ & $7 \pm 2$ & $1.33 \pm 0.05$ \\
E267A & $96 \pm 2$ & $91 \pm 6$ & $9 \pm 1$ & $28.3 \pm 0.9$ & $10 \pm 2$ & $1.42 \pm 0.03$ \\
K269A & $42 \pm 2$ & $310 \pm 40$ & $25 \pm 6$ & $89 \pm 7$ & $12 \pm 4$ & $1.39 \pm 0.08$ \\
K269R & $51 \pm 2$ & $122 \pm 12$ & $32 \pm 8$ & $63 \pm 6$ & $4 \pm 1$ & $1.23 \pm 0.06$ \\
K269E & $62 \pm 2$ & $144 \pm 15$ & $31 \pm 6$ & $70 \pm 6$ & $5 \pm 1$ & $1.26 \pm 0.06$ \\
R295A & $\mathbf{2 . 7} \pm \mathbf{0 . 4}$ & $320 \pm 40$ & $\mathbf{1 3 0} \pm \mathbf{7 0}$ & $\mathbf{1 9 0} \pm \mathbf{4 0}$ & $2.6 \pm 1.5$ & $1.25 \pm 0.11$ \\
R295L & $\mathbf{3 . 8} \pm \mathbf{0 . 1}$ & $34 \pm 5$ & $2.3 \pm 0.4$ & $9.0 \pm 0.4$ & $15 \pm 5$ & $1.50 \pm 0.05$ \\
R295E & $43 \pm 3$ & $\mathbf{1 2 0 0} \pm \mathbf{2 0 0}$ & $\mathbf{6 0} \pm \mathbf{1 6}$ & $\mathbf{2 7 0} \pm \mathbf{3 0}$ & $20 \pm 7$ & $1.43 \pm 0.10$ \\
N312A & $\mathbf{0 . 7 3} \pm \mathbf{0 . 0 1}$ & $5.6 \pm 0.2$ & $5.4 \pm 0.2$ & $5.52 \pm 0.08$ & $\mathbf{1 . 0 5} \pm \mathbf{0 . 0 7}$ & $\mathbf{1 . 0 1} \pm \mathbf{0 . 0 2}$ \\
R334A & $\mathbf{1 1} \pm \mathbf{1}$ & $40 \pm 5$ & $37 \pm 3$ & $39 \pm 1$ & $\mathbf{1 . 0} \pm \mathbf{0 . 1}$ & $\mathbf{1 . 0 2} \pm \mathbf{0 . 1 0}$ \\
R334K & $52 \pm 2$ & $78 \pm 8$ & $33 \pm 8$ & $50 \pm 5$ & $2.3 \pm 0.6$ & $1.14 \pm 0.07$ \\
R334E & $<0.2$ & & & & & \\
\hline Substrate & & & & & & \\
\hline
\end{tabular}

a Substrate concentration was varied in the range of $0.5-500 \mu \mathrm{M}$.

${ }^{b}$ From reference 3 ; the $K_{\mathrm{m}}$ values were converted to microscopic constants. 
Table S7. Parameter values in Scheme 1 for $\mathrm{PP}_{\mathrm{i}}$ hydrolysis by $d h \mathrm{PPase}$ variants measured in the presence of AMP at $5 \mathrm{mM}$ free $\mathrm{Mg}^{2+}$. Large effects are indicated by boldface.

\begin{tabular}{lccccccc}
\hline Enzyme & $\begin{array}{c}\mathrm{AMP}, \\
\mathrm{MM}\end{array}$ & $k_{\mathrm{cat},}, \mathrm{s}^{-1}$ & $K_{\mathrm{m} 1}, \mu \mathrm{M}$ & $K_{\mathrm{m} 2}, \mu \mathrm{M}$ & $\begin{array}{c}\sqrt{K_{\mathrm{m} 1} K_{\mathrm{m} 2},} \\
\mu \mathrm{M}\end{array}$ & $K_{\mathrm{m} 1} / K_{\mathrm{m} 2}$ & $h$ \\
\hline Wild type $^{a}$ & 50 & $1.31 \pm 0.03$ & $106 \pm 12$ & $30 \pm 5$ & $57 \pm 3$ & $3.5 \pm 1.0$ & $1.27 \pm 0.07$ \\
S266A & 50 & $10.2 \pm 0.3$ & $220 \pm 20$ & $29 \pm 5$ & $81 \pm 4$ & $8 \pm 2$ & $1.35 \pm 0.06$ \\
E267A & 50 & $2.9 \pm 0.2$ & $36 \pm 3$ & $100 \pm 40$ & $60 \pm 12$ & $\mathbf{0 . 4} \pm \mathbf{0 . 1}$ & $\mathbf{0 . 8 0} \pm \mathbf{0 . 0 2}$ \\
K269A & 50 & $1.92 \pm 0.04$ & $75 \pm 4$ & $25 \pm 3$ & $44 \pm 2$ & $3.0 \pm 0.5$ & $1.19 \pm 0.04$ \\
R295A & 50 & $0.60 \pm 0.05$ & $14 \pm 2$ & $130 \pm 70$ & $43 \pm 15$ & $\mathbf{0 . 1 0} \pm \mathbf{0 . 0 4}$ & $\mathbf{0 . 4 6} \pm \mathbf{0 . 0 4}$ \\
R295L & 50 & $1.17 \pm 0.02$ & $31 \pm 1$ & $9.7 \pm 0.8$ & $17.3 \pm 0.5$ & $3.1 \pm 0.4$ & $1.22 \pm 0.03$ \\
R295E & 500 & $3.3 \pm 0.2$ & $76 \pm 5$ & $71 \pm 20$ & $73 \pm 10$ & $1.1 \pm 0.3$ & $0.97 \pm 0.04$ \\
N312A & 50 & $0.170 \pm 0.002$ & $\mathbf{2 . 4} \pm \mathbf{0 . 2}$ & $\mathbf{3 . 5} \pm \mathbf{0 . 3}$ & $\mathbf{2 . 9 0} \pm \mathbf{0 . 0 9}$ & $0.69 \pm 0.09$ & $0.91 \pm 0.03$ \\
R334A & 500 & $0.41 \pm 0.02$ & $19 \pm 2$ & $47 \pm 14$ & $30 \pm 4$ & $\mathbf{0 . 4} \pm \mathbf{0 . 1}$ & $\mathbf{0 . 7 6} \pm \mathbf{0 . 0 5}$ \\
\hline
\end{tabular}

${ }^{a}$ From reference 4.

Table S8. Parameter values in Scheme 1 for $\mathrm{PP}_{\mathrm{i}}$ hydrolysis by $d h \mathrm{PPase}$ variants measured in the presence of $5 \mu \mathrm{M} \mathrm{Ap} \mathrm{A}_{4} \mathrm{~A}$ at $5 \mathrm{mM}$ free $\mathrm{Mg}^{2+}$. Large effects of the mutations are indicated by boldface.

\begin{tabular}{lcccccc}
\hline Enzyme & $k_{\text {cat }}, \mathrm{s}^{-1}$ & $K_{\mathrm{m} 1}, \mu \mathrm{M}$ & $K_{\mathrm{m} 2}, \mu \mathrm{M}$ & $\begin{array}{c}\sqrt{ } K_{\mathrm{m} 1} K_{\mathrm{m} 2}, \\
\mu \mathrm{M}\end{array}$ & $K_{\mathrm{m} 1} / K_{\mathrm{m} 2}$ & $h$ \\
\hline Wild type & $310 \pm 10$ & $7 \pm 0.6$ & $7 \pm 2$ & $7 \pm 2$ & $0.95 \pm 0.08$ & $1.00 \pm 0.05$ \\
S266A & $157 \pm 2$ & $3.8 \pm 0.3$ & $2.4 \pm 0.2$ & $3.0 \pm 0.1$ & $1.6 \pm 0.3$ & $1.10 \pm 0.04$ \\
S266D & $206 \pm 2$ & $3.3 \pm 0.2$ & $1.9 \pm 0.1$ & $2.49 \pm 0.06$ & $1.7 \pm 0.2$ & $1.13 \pm 0.03$ \\
E267A & $185 \pm 4$ & $2.9 \pm 0.4$ & $2.8 \pm 0.4$ & $2.8 \pm 0.2$ & $1.0 \pm 0.3$ & $1.00 \pm 0.06$ \\
K269A & $120 \pm 2$ & $3.3 \pm 0.2$ & $3.7 \pm 0.3$ & $3.5 \pm 0.1$ & $0.9 \pm 0.1$ & $0.98 \pm 0.03$ \\
K269R & $218 \pm 3$ & $2.3 \pm 0.2$ & $2.3 \pm 0.2$ & $2.3 \pm 0.1$ & $1.0 \pm 0.1$ & $1.00 \pm 0.03$ \\
K269E & $157 \pm 3$ & $3.6 \pm 0.3$ & $3.6 \pm 0.4$ & $3.6 \pm 0.1$ & $1.0 \pm 0.2$ & $1.00 \pm 0.04$ \\
R295A & $117 \pm 3$ & $>\mathbf{1 0 0 0}$ & $<1$ & $33 \pm 1$ & $>\mathbf{1 0 0 0}$ & $\mathbf{2 . 2 4} \pm \mathbf{0 . 1 2}$ \\
R295L & $48 \pm 1$ & $>\mathbf{1 0 0 0}$ & $<1$ & $22.4 \pm 0.4$ & $>\mathbf{1 0 0 0}$ & $\mathbf{2 . 0 1} \pm \mathbf{0 . 0 3}$ \\
R295E & $145 \pm 4$ & $18 \pm 2$ & $3.0 \pm 0.4$ & $7.4 \pm 0.3$ & $\mathbf{6 . 3} \pm \mathbf{1 . 5}$ & $\mathbf{1 . 3 6} \pm \mathbf{0 . 0 5}$ \\
N312A & $\mathbf{1 0 . 2} \pm \mathbf{0 . 2}$ & $\mathbf{1 1 0} \pm \mathbf{4 0}$ & $1.4 \pm 0.5$ & $15.1 \pm 0.1$ & $\mathbf{8 0} \pm \mathbf{6 0}$ & $\mathbf{1 . 7 9} \pm \mathbf{0 . 0 8}$ \\
R334A & $64 \pm 2$ & $2.9 \pm 0.5$ & $0.8 \pm 0.2$ & $1.50 \pm 0.07$ & $\mathbf{4} \pm \mathbf{1}$ & $\mathbf{1 . 3 0} \pm \mathbf{0 . 0 7}$ \\
R334K & $259 \pm 2$ & $5.9 \pm 0.5$ & $\mathbf{0 . 6 2} \pm \mathbf{0 . 0 5}$ & $1.91 \pm 0.03$ & $\mathbf{9 . 6} \pm \mathbf{1 . 7}$ & $\mathbf{1 . 4 8} \pm \mathbf{0 . 0 3}$ \\
\hline
\end{tabular}


Table S9. Parameter values in Scheme 1 for $\mathrm{PP}_{\mathrm{i}}$ hydrolysis by $d h \mathrm{PPase}$ variants measured in the presence of ATP at $5 \mathrm{mM}$ free $\mathrm{Mg}^{2+}$. Large effects of the mutations are indicated by boldface.

\begin{tabular}{lccccccc}
\hline Enzyme & {$[\mathrm{ATP}], \mu \mathrm{M}$} & $k_{\text {cat }}, \mathrm{s}^{-1}$ & $K_{\mathrm{m} 1}, \mu \mathrm{M}$ & $K_{\mathrm{m} 2}, \mu \mathrm{M}$ & $\begin{array}{c}\sqrt{ } K_{\mathrm{m} 1} K_{\mathrm{m} 2}, \\
\mu \mathrm{M}\end{array}$ & $K_{\mathrm{m} 1} / K_{\mathrm{m} 2}$ & $h$ \\
\hline Wild type $^{a}$ & 10 & $225 \pm 5$ & $20 \pm 2$ & $5.5 \pm 0.5$ & $10 \pm 1$ & $3.7 \pm 0.1$ & $1.24 \pm 0.02$ \\
$\mathrm{R} 295 \mathrm{~A}$ & 100 & $\mathbf{0 . 6 5} \pm \mathbf{0 . 0 5}$ & $32 \pm 4$ & $130 \pm 60$ & $60 \pm 20$ & $\mathbf{0 . 2 5} \pm \mathbf{0 . 1 1}$ & $\mathbf{0 . 8 5} \pm \mathbf{0 . 0 4}$ \\
$\mathrm{R} 295 \mathrm{~L}$ & 100 & $\mathbf{1 . 4 4} \pm \mathbf{0 . 0 4}$ & $38 \pm 3$ & $16 \pm 2$ & $24.5 \pm 1.5$ & $2.4 \pm 0.5$ & $1.17 \pm 0.04$ \\
$\mathrm{~N} 312 \mathrm{~A}$ & 100 & $\mathbf{0 . 9 6} \pm \mathbf{0 . 0 5}$ & $\mathbf{4 4 0} \pm \mathbf{3 0}$ & $\mathbf{1 4 0} \pm \mathbf{3 0}$ & $\mathbf{2 5 0} \pm \mathbf{2 0}$ & $3.0 \pm 0.7$ & $1.15 \pm 0.05$ \\
\hline
\end{tabular}

${ }^{a}$ From reference 3 ; the $K_{\mathrm{m}}$ values were converted to microscopic constants.

\section{References}

1. Crooks, G.E., Hon, G., Chandonia, J.M. and Brenner, S.E. (2004) WebLogo: A sequence logo generator. Genome Research 14, 1188-1190

2. Anashkin, V. A.; Salminen, A.; Tuominen, H. K.; Lahti, R.; Baykov, A. A. Cystathionine $\beta$-synthase (CBS) domain-containing pyrophosphatase as a target for diadenosine polyphosphates in bacteria. $J$. Biol. Chem. 2015, 290, 27594-27603.

3. Salminen, A., Anashkin, V.A., Lahti, M., Tuominen, H.K., Lahti, R. and Baykov, A.A. (2014) Cystathionine $\beta$-synthase (CBS) domains confer multiple forms of $\mathrm{Mg}^{2+}$-dependent co-operativity to Family II pyrophosphatases. J. Biol. Chem. 289, 22865-22876

4. Anashkin, V.A., Orlov, V.N., Lahti R. and Baykov A.A. (2019) An arginine residue involved in allosteric regulation of cystathionine $\beta$-synthase (CBS) domain-containing pyrophosphatase. Arch. Biochem. Biophys. 662, 40-48 